\title{
Editorial
}

\section{Materials for Nuclear and Fossil Energy Applications}

\author{
Kisor Kumar Sahu, ${ }^{1,2}$ Ajit Kumar Roy, ${ }^{1}$ Amritendu Roy, ${ }^{1}$ \\ Kaushik Das, ${ }^{1}$ and Randhir Singh ${ }^{1}$ \\ ${ }^{1}$ School of Minerals, Metallurgical and Materials Engineering, Indian Institute of Technology Bhubaneswar, Bhubaneswar 751007, India \\ ${ }^{2}$ Center of Excellence for Novel Energy Materials (CENEMA), Indian Institute of Technology Bhubaneswar, Bhubaneswar 751007, India
}

Correspondence should be addressed to Kisor Kumar Sahu; kisorsahu@iitbbs.ac.in

Received 18 January 2016; Accepted 19 January 2016

Copyright ( 2016 Kisor Kumar Sahu et al. This is an open access article distributed under the Creative Commons Attribution License, which permits unrestricted use, distribution, and reproduction in any medium, provided the original work is properly cited.

We are extremely happy to release this special issue. Energy is the prime driver for modern civilization. Strong inverse correlation between per capita energy consumption and poverty level suggests that, in order to alleviate poverty and enhancement of general living standards, the global energy consumption will grow manyfold in the coming decades. With presentday technologies, this is simply not feasible particularly for the choice of present-day resource portfolio and the associated economic cost of scaling. For example, according to one estimate, for the global population to achieve western European living standard, characterized by moderate rate of per capita energy consumption $(\approx 7 \mathrm{~kW}$ compared to $\approx 11 \mathrm{~kW}$ for USA), we will need $\$ 2.2$ trillion per year investment for next 20 years in a sustained rate at present-day prices. While one can debate about these numbers, what is clear is that, without technological advancements and breakthroughs, majority of the global population will have no choice but to settle for very poor living standards. Global climate change and deteriorating air quality in urban areas are also a direct consequence of how we source and utilize energy. Since the growth rate of cleaner energy sources is far lower than needed, serious technical research is necessary for better utilization of the traditional resources. Generally, materials are the bottleneck in developing new methods and technologies and hence, in this issue, we have tried to encompass a broad range of topics related to the materials aspects of nuclear energy-based and fossil fuel-based energy generation.

Articles presented in this issue are related to Sodiumcooled Fast Reactors (SFRs), Very High Temperature gascooled Reactors (VHTRs), Pressurized Water Reactors
(PWRs), and other reactors. Alloy development for nuclear fuel rods is an important topic in nuclear technology, and in this issue the article by J.-H. Kim and coworkers discusses the development of an injection casting process for preparing fuel slugs of U-10Zr-5Mn, a surrogate alloy for use in Sodiumcooled Fast Reactors (SFRs). Alloys to be used in energy generation plants have stringent requirements in terms of high temperature mechanical properties. In this issue, S. J. Kim and coworkers have presented a comparison of cyclic stress response and fracture behavior of Alloy 617 in the form of base metal and in the form of welded joints. Another critical issue in nuclear power generation is corrosion inhibition of the inner linings of the pipes and the tubes. These alloys get corroded due to exposure to primary water of nuclear power plants. Surface treatment of the alloys and/or inhibitor addition to the primary water are two approaches to inhibit corrosion, and these approaches are discussed in the articles by K. M. Kim et al. and by S. J. Kim et al., respectively.

Materials challenges in nuclear waste management and in recovery of nuclear fuel from spent fuel rods have also been considered in this issue. In addition, design of new generation of low power thorium-based nuclear technology also forms a part of this issue.

Kisor Kumar Sahu Ajit Kumar Roy Amritendu Roy Kaushik Das Randhir Singh 

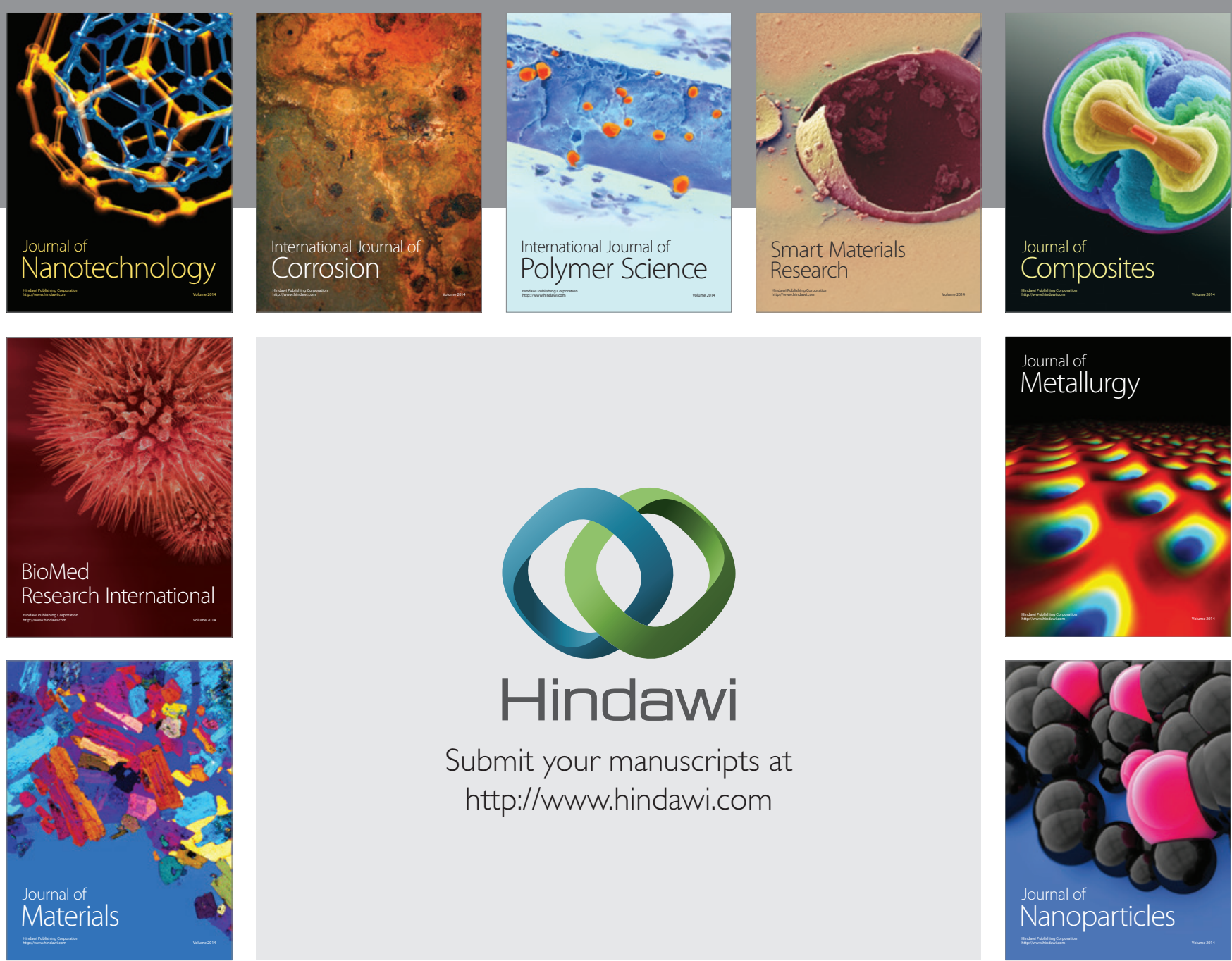

\section{Hindawi}

Submit your manuscripts at

http://www.hindawi.com

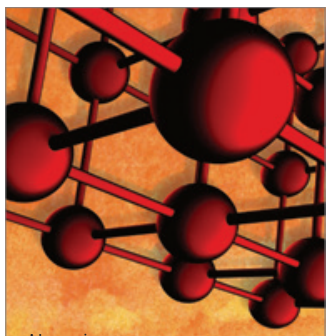

Materials Science and Engineering
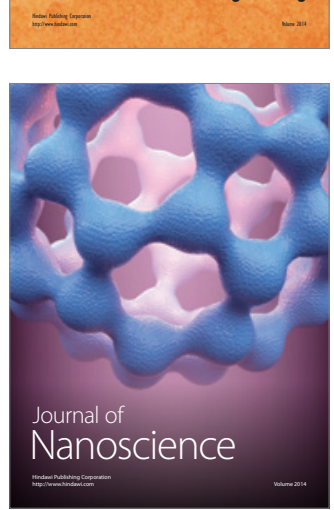
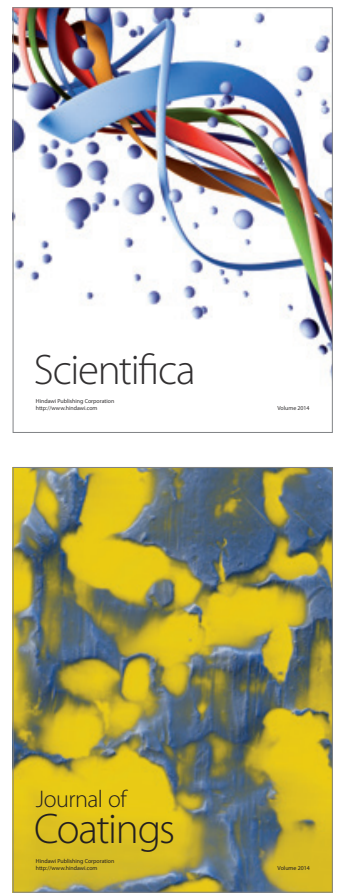
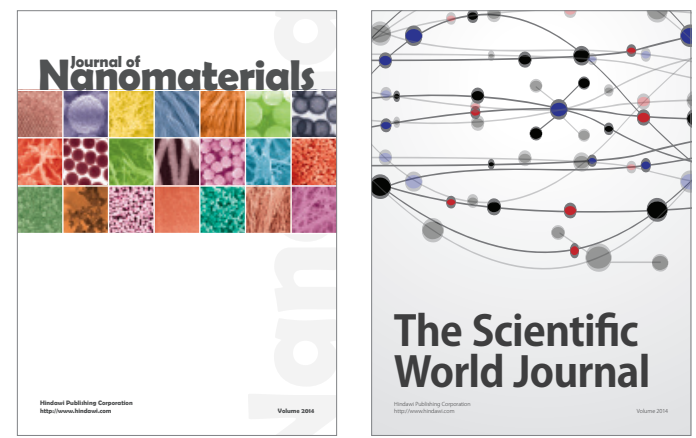

The Scientific World Journal
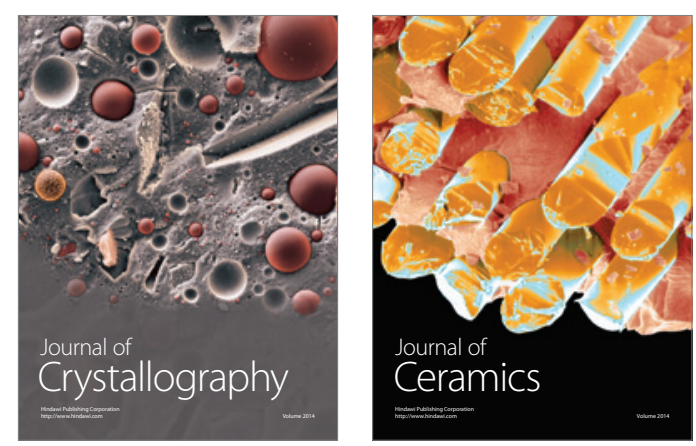
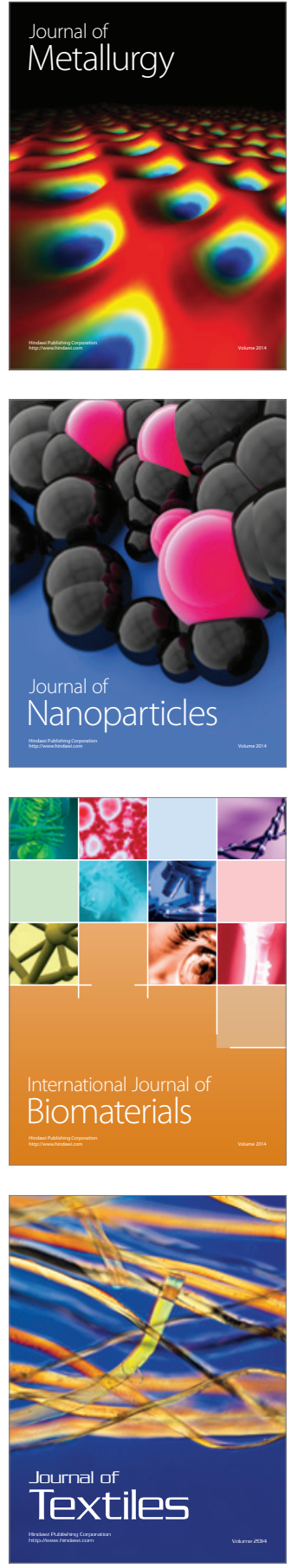